\section{La Révolution française}

Cahiers de l'Institut d'histoire de la Révolution française

$4 \mid 2013$

Pédagogies, utopies et révolutions (1789-1848)

\title{
Tableaux synoptiques et récits historiques : La référence aux modèles étrangers dans le cours d'histoire des Écoles centrales (1795-1802)
}

Pauline Pujo

\section{OpenEdition}

\section{Journals}

Édition électronique

URL : http://journals.openedition.org//rf/834

DOI : $10.4000 / / r f .834$

ISSN : 2105-2557

Éditeur

IHMC - Institut d'histoire moderne et contemporaine (UMR 8066)

Référence électronique

Pauline Pujo, «Tableaux synoptiques et récits historiques : La référence aux modèles étrangers dans le cours d'histoire des Écoles centrales (1795-1802) », La Révolution française [En ligne], 4 | 2013, mis en ligne le 10 juin 2013, consulté le 19 avril 2019. URL : http://journals.openedition.org//rf/834 ; DOI 10.4000/lrf.834

Ce document a été généré automatiquement le 19 avril 2019

(C) La Révolution française 


\title{
Tableaux synoptiques et récits historiques : La référence aux modèles étrangers dans le cours d'histoire des Écoles centrales
} (1795-1802)

\author{
Pauline Pujo
}

\section{Les modèles étrangers : une approche nouvelle pour l'étude de la pédagogie révolutionnaire}

1 Si l'importance historique des projets pédagogiques de la Révolution a été démontrée et située dans la continuité de l'histoire nationale, le rôle des modèles pédagogiques étrangers dans l'élaboration d'une pédagogie dite républicaine reste encore aujourd'hui peu exploré ${ }^{1}$. Pourtant, la singularité de l'histoire politique française n'a pas rendu les frontières hermétiques, pas plus qu'elle n'a fait table rase de l'héritage pédagogique de l'Ancien Régime et des Lumières's . Le recours à des modèles pédagogiques anglais et allemands peut même s'expliquer par un rapport problématique aux manuels et aux méthodes d'avant 1789 encore en usage dans les Écoles centrales créées par les lois du 7 ventôse an III (25 février 1795) et du 3 brumaire an IV (25 octobre 1795) et supprimées par la loi du 11 floréal an X ( $1^{\mathrm{er}}$ mai 1802). Il s'inscrit dans un contexte de redéfinition des modèles de référence en histoire et en pédagogie, et dans un contexte de politisation de la pédagogie de l'histoire, auparavant affectée à la formation de l'homme d'État, et dorénavant destinée à la formation du citoyen ${ }^{3}$. S'intéresser aux modèles étrangers permet ainsi de s'affranchir du prisme projet-réalisation à travers lequel la pédagogie révolutionnaire a pu être perçue, pour aborder les Écoles centrales comme un terrain sur lequel s'affrontent plusieurs modèles concurrents de la pédagogie de l'histoire ${ }^{4}$. 
2 Pierre-Jean Massenet, professeur d'histoire à l'École centrale du Bas-Rhin et PierreNicolas Chantreau, professeur d'histoire à l'École centrale du Gers promeuvent un mode tabulaire de présentation des connaissances historiques, sous forme de tablettes chronologiques, de tableaux synoptiques croisant l'axe du temps en abscisse et de l'espace en ordonnée, ou sous forme de nomenclature des différentes branches de la science historique. Ces deux professeurs se réfèrent à des modèles pédagogiques étrangers (allemand pour Massenet, anglais pour Chantreau) pour légitimer leur position et s'opposer aux membres du Conseil d'instruction publique qui restent fidèles aux longues narrations de l'histoire universelle héritées des Lumières. En utilisant des chronologies et des tableaux synoptiques, Massenet et Chantreau n'innovent aucunement et auraient pu trouver des modèles en France. Bien loin d'être une invention des Lumières, les tableaux sont en effet une constante de l'historiographie européenne moderne ${ }^{5}$. Le choix délibéré de références étrangères, dicté par des enjeux sociaux et géographiques, n'en affecte pas moins la pédagogie de l'histoire en elle-même. Le tableau auparavant utilisé en complémentarité du récit, va être présenté comme une méthode alternative et opposée au récit. Ce qui n'était qu'un outil de travail utilisé en complémentarité avec d'autres formes de représentation de l'histoire devient, par le jeu des références aux modèles étrangers et par démarcation face au pouvoir central, une méthode pédagogique à part entière, utilisée par opposition à d'autres méthodes.

Un exemple de présentation tabulaire du savoir historique : Pierre-Nicolas CHANTREAU, La Science de I'histoire, t. 1 : Chronologie, Paris, Goujon, an XI/1803, Discours préliminaire, VII. 


\section{La pédagogie du tableau entre voir et savoir}

\section{Les contours d'un genre historiographique}

Avant de comprendre comment les deux professeurs ont pu associer le tableau synoptique à une méthode particulière, il est nécessaire de définir les contours de ce genre historique : la présentation sous forme de liste ou de tableau constitué de lignes et de colonnes, présentation synthétique des informations qui permet de toutes les embrasser d'un coup d'oeil, d'en ajouter et de les comparer ${ }^{6}$. Dans ce genre historiographique fondé sur la compilation des informations, la figure de l'auteur est fortement effacée, ce qui explique la longévité de certains tableaux, remaniés par leurs différents usagers au fil des siècles, et ce qui demande une lecture active, constructive, interprétative. Pour lire un tableau aux élèves, le professeur doit lier les informations qu'il y trouve, produire lui-même un récit ou une interprétation historique, choisir un angle d'attaque et un questionnement particulier pour entrer dans le tableau. La valorisation de la vue dans la transmission des connaissances n'est en rien l'apanage de la modernité, mais elle constitue un topos antique qui traverse le Moyen-Âge et la Renaissance jusqu'au Lumières ${ }^{7}$. Ainsi, quand Chrétien-Siméon Le Prévost D'Iray, professeur d'histoire à l'École de la rue Saint-Antoine, souligne les vertus pédagogiques des tableaux synoptiques qu'il a composés, il se situe à la fois dans la continuité de l' Encyclopédie de Diderot et D'Alembert et d'une longue tradition européenne :

«[l'élève] voit d'un coup d'oeil, sans être plus distrait, ce qui se passe aux extrêmités du globe ; saisit l'enchaînement des révolutions qui paraissent les plus étrangères l'une à l'autre; et, dans une sorte d'étonnement perpétuel, démêlant entre tout ce qu'il voit à la fois, les rapports les plus intimes, se sent attaché avec plus de force à sa lecture et y revient avec transport ${ }^{8}$. "

De plus, le tableau est une forme complémentaire à d'autres formes de transmission de l'histoire. En gardant les mêmes structures et les mêmes informations, il est possible de présenter la même chose sous forme de catéchisme historique, sous forme de tableau, sous forme de récit ou sous forme d'image. De même, il est possible de faire un usage parallèle et complémentaire des récits historiques et des tableaux synoptiques. Enfin, un tableau ne renvoie pas à une forme de lecture ou à une méthode pédagogique précise, mais peut aussi bien servir de répertoire à mémoriser pour le débutant que de système d'orientation et de localisation des informations pour l'érudit.

\section{Tableau et élément dans la production des savoirs (XVII ${ }^{\mathrm{e}} \mathrm{XVIII}{ }^{\mathrm{e}}$ siècles)}

Bien loin d'être limité à la pédagogie de l'histoire, le tableau occupe une place centrale dans la production des savoirs aux XVIIe et xvire siècles et repose sur un rapport particulier entre voir et savoir ${ }^{9}$. Il marque une rupture avec la Renaissance, où savoir consistait à trouver des similitudes parmi les choses du monde - voir à lire et interpréter les signes que portaient les choses pour marquer ces similitudes. Le célèbre ouvrage du pédagogue tchèque Comenius, l'Orbis sensualium pictus (1658), tient de cette tradition proche de l'emblème, qui fait primer l'écrit sur la vue, révèle les mots qui correspondent à chaque objet et fait se refléter le macrocosme ("Orbis») dans le microcosme de l'enfant : il présente une série d'images assorties de listes de mots désignant les objets des 
images en plusieurs langues ${ }^{10}$. Chez Comenius, les sens ne sont que des canaux qui permettent à l'enfant d'acquérir plus facilement une connaissance fondamentalement livresque. Cependant, l'Orbis sensualium pictus est généralement considéré dans l'histoire de la pédagogie comme une étape vers le sensualisme qui, de John Locke à Jean-Jacques Rousseau, fait des sens des agents à part entière de la connaissance ${ }^{11}$. Dans la production des savoirs à l'époque classique, l'écrit perd en effet sa prééminence et cède la place à la vue. Si, désormais, les idées viennent des sens, on met cependant de côté le toucher, le goût, l'ouïe et l'odorat pour se centrer sur la vue comme expérience concrète qui pointe vers l'abstraction parce qu'elle s'effectue à distance des objets observés et permet d'ordonner les choses dans un système d'identités et de différences ${ }^{12}$. Ainsi s'effectue le glissement de l'observation du monde vers la contemplation du tableau dont on ne se lasse pas de souligner qu'il permet de tout voir d'un coup, de synthétiser les connaissances dans un panorama. La vue est certes source de savoir empirique, mais elle aide surtout à penser, elle permet à l'esprit de prendre de la hauteur, d'accéder à une vision d'ensemble - un tableau synoptique - que la seule raison ne pourrait atteindre.

Dans le sillage de la pensée classique, l'Encyclopédie accorde, elle aussi, une place centrale au tableau dans la production du savoir, notamment à travers son Système Figuré des Connaissances Humaines et son Essai d'une distribution généalogique des Sciences et des Arts Principaux. Dans le Discours préliminaire et l'article "Élémens des sciences» de l' Encyclopédie ainsi que dans son Essai sur les éléments de philosophie, D'Alembert élabore une conception dynamique de la notion d'élément et établit entre élément et tableau des liens problématiques qui nourriront quelques années plus tard la réflexion de Condorcet sur les savoirs élémentaires dans la pédagogie républicaine ${ }^{13}$. À l'ordre chronologique de la découverte des savoirs et des techniques, dépendant de facteurs contingents tels que les besoins et les plaisirs des hommes, D'Alembert oppose l'ordre rationnel exposé dans le Système Figuré des Connaissances Humaines. Se fier à l'ordre des découvertes, c'est entrer dans un labyrinthe où on ne saisit pas les liens qui relient les savoirs entre eux. Se rapporter au tableau, c'est se placer au-dessus du labyrinthe et élaborer une cartographie des connaissances pour s'y orienter. Mais cet effort d'orientation ne peut être lui-même objet de savoir et être érigé en science fondamentale, d'une part parce que ce serait trouver un substitut à la théologie que le Système a détrôné de cette position souveraine et d'autre part parce que le dessin de la carte est une projection faite à partir d'un point de vue particulier. Ni l'ordre des découvertes ni la cartographie du tableau ne constituent un principe de rationalité. D'Alembert le situe au contraire dans une conception dynamique de la notion d'élément. L'élément est à la fois le produit d'une réflexion qui remonte des savoirs acquis au fil du temps vers leurs principes, des origines contingentes aux origines nécessaires. Il est à la fois le point de départ de progrès scientifiques et techniques à venir, nouvelle origine dans l'ordre des découvertes, conçue d'abord comme nécessaire mais que le progrès révèle comme contingente ${ }^{14}$.

De cette relation dialectique entre éléments et tableau, qui mêle une conception linéaire à une conception cyclique du temps, Condorcet retient en particulier la dimension émancipatrice ${ }^{15}$. À la notion d'éléments des sciences, il ajoute la notion d'éléments scolaires, forme didactisée de ces derniers. Dans une pédagogie républicaine, le savoir élémentaire est à la fois ce qui peut servir à tout citoyen (lire, écrire, compter, comprendre les lois) et ce qui renvoie au tableau général des connaissances, garantissant ainsi doublement l'autonomie de chacun. On ne dépend pas de plus instruit que soi pour payer ses impôts par exemple. On ne dépend pas non plus des savants pour situer les 
savoirs élémentaires que l'on possède dans le tableau général des connaissances ${ }^{16}$. Une telle définition des savoirs élémentaires entraîne une opposition entre élémentaire et abrégé, que Joseph Lakanal formule avec concision en l'an III : «présenter les premiers germes et en quelque sorte la matrice d'une science, c'est l'élémenter : ainsi l'abrégé, c'est précisément le contraire de l'élémentaire ${ }^{17}$.» Alors que l'élémentaire suppose une continuité entre le savoir du simple citoyen et celui des savants, l'abrégé, fragment de connaissances sans lien avec le système qu'elles forment, instaure une relation inégale de dépendance entre le maître et l'élève.

Dans l'écriture et la pédagogie de l'histoire, on oppose de même l'agrégat au système, la chronique qui amasse les faits selon un ordre contingent (les besoins de légitimation du pouvoir notamment) à l'histoire philosophique qui recherche les causes liant les faits entre eux dans un ordre nécessaire. Élémentaire et abrégé, système et agrégat amènent ainsi à repenser des modes de représentation de l'histoire héritées du passé, comme le tableau et la narration, et à leurs attribuer une signification nouvelle. Par le jeu des références à des modèles pédagogiques étrangers, Massenet et Chantreau en viennent ainsi à situer le tableau du côté de l'élémentaire et la narration du côté de l'abrégé.

De telles associations ne sont pas nécessaires. D'autres études ont montré comment la narration pouvait au contraire être le support d'une historiographie qui lie les faits entre eux, qui entrecroise l'ordre logique et l'ordre chronologique et, par le récit d'un simple fait, renvoie également à un système plus vaste ${ }^{18}$. Tout dépend de l'interprétation et du nouvel usage que l'on souhaite faire des formes historiographiques alors disponibles. Le fait que Massenet et Chantreau opposent tableau et narration, qu'ils associent le tableau à une méthode pédagogique et un arrière-plan politique particulier, peut s'expliquer dans un premier temps par un rapport problématique à l'héritage de l'Ancien Régime.

\section{Quels modèles français pour l'historiographie républicaine?}

\section{L'historiographie des Lumières, un héritage mal assumé}

C'est plus précisément la question des livres élémentaires qui représente une pierre d'achoppement dans les débats sur l'enseignement républicain de l'histoire. Dans la continuité du concours du 9 pluviôse an II (28 janvier 1794), les membres du Comité puis Conseil d'instruction publique du Directoire vont s'attacher à contrôler, puis à favoriser l'élaboration de livres élémentaires qui permettent l'uniformisation de l'enseignement et la formation de maitres peu expérimentés ou habitués à la pédagogie d'Ancien Régime ${ }^{19}$. Une série de circulaires envoyées les 20 fructidor an V (6 septembre 1797), 7 vendémiaire an VII (28 septembre 1798) et le 5e jour complémentaire an VII (21 septembre 1799), demande aux professeurs d'envoyer les cahiers ou le plan de leurs cours à l'administration centrale en vue de l'élaboration de livres élémentaires. Bien loin d'être relégués au rang de simples exécutants d'un projet pédagogique émanant du législateur, les professeurs sont au contraires appelés à élaborer une pédagogie nouvelle en même temps qu'ils l'expérimentent, et c'est dans la réponse de Pierre-Jean Massenet et Pierre-Nicolas Chantreau à ces circulaires que l'on voit s'opposer des modèles pédagogiques concurrents. 
11 La question des livres élémentaires est en effet traversée d'une double opposition: d'une part l'opposition entre éléments et abrégé, évoquée plus haut, et d'autre part l'opposition entre savoirs républicains et royalistes qui se manifeste en particulier à la suite du coup d'État du 18 fructidor an V (4 septembre 1797) ${ }^{20}$. L'envoi de la première circulaire aux professeurs des Écoles centrales date en effet du surlendemain de l'événement et est suivie d'une série de rapports sur des livres élémentaires d'histoire royalistes ou considérés comme tels parce qu'ils ne se réfèrent pas assez explicitement au régime républicain ${ }^{21}$.

De cette double opposition résulte un rapport problématique à la pédagogie et à l'historiographie d'avant 1789. D'une part, la référence à l'élémentation situe la pédagogie révolutionnaire dans la continuité des Lumières. D'autre part, cette continuité reste problématique parce qu'elle suppose également une continuité avec l'Ancien Régime. Dans l'élaboration de méthodes et de contenus d'enseignement, dans la quête de livres élémentaires républicains, ce qu'il faut observer n'est donc pas tant la création d'une pédagogie révolutionnaire radicalement nouvelle, mais en premier lieu l'évolution des modèles de référence. C'est en cela que l'étude des modèles étrangers s'avère intéressante.

\section{Les Élémens d'histoire générale de l'abbé Millot (1772-1773) : une référence problématique}

13 Le contrôle des livres d'histoire à la suite du 18 fructidor an V (4 septembre 1797) est une bonne illustration du statut spécifique de cette matière en comparaison, par exemple, avec la grammaire générale, également enseignée dans les Écoles centrales. Une histoire républicaine doit se construire à la fois par emprunt et par opposition à l'historiographie antérieure alors qu'il n'existe pas de grammaire générale royaliste ou chrétienne dont une grammaire générale républicaine aurait à se démarquer.

Ce rapport problématique de l'histoire à l'héritage de l'Ancien Régime et des Lumières se retrouve également dans l'élaboration - inaccomplie - d'un livre élémentaire d'histoire par le Conseil d'instruction publique créé le 11 brumaire an VII (1er novembre 1798) et placé sous la tutelle de l'idéologue Destutt de Tracy ${ }^{22}$. L'un des cahiers envoyés par un professeur en réponse aux circulaires est une version abrégée des Élemens d'histoire générale, longue narration de l'histoire universelle, composée dans un esprit voltairien par l'abbé François-Xavier Millot (1726-1785) pour l'éducation de la noblesse de Parme, au début des années 1770, puis utilisée à l'École royale militaire et traduite dans plusieurs langues européennes ${ }^{23}$. Le Conseil, qui cherche dans les cahiers des professeurs l'embryon d'un nouveau livre élémentaire, se réjouit dans un premier temps de cet envoi. Un rapport élogieux est rédigé. On se rend compte peu après qu'il s'agit d'une version épurée du livre de Millot et une note en marge exprime la déception du rapporteur. Cette déconvenue, et la réticence du Conseil à assumer la filiation avec la pédagogie de l'histoire de Millot, apporte cependant un aperçu précieux du livre élémentaire visé. Il s'agirait d'une longue narration de l'histoire universelle, plus courte que celle de Millot, probablement laïcisée.

À quel usage pédagogique ce livre élémentaire était-il destiné ? À une lecture solitaire par chaque élève, dans la bibliothèque de l'école ou à la maison, puis à une relecture commentée en cours, invitant à la comparaison entre les peuples, entre les événements, entre les époques. La circulaire envoyée par Destutt de Tracy le 5e jour complémentaire 
an VII (21 septembre 1799) décrit cet usage, qui doit notamment remplacer celui de la dictée (si la dictée du cours est généralement rejetée par les professeurs, elle est en effet pratiquée, notamment en raison de la pénurie de livres élémentaires ${ }^{24}$ ). Le Conseil d'instruction publique du Directoire promeut un usage des livres élémentaires qui fait de la médiation du professeur un élément essentiel de l'accès à la connaissance historique. Il va à l'encontre de la conception alors largement répandue selon laquelle il suffit de lire l'histoire pour la connaitre, conception qui repose sur le lien traditionnel entre histoire et rhétorique ${ }^{25}$.

\section{Le cours d'Edme Mentelle (1730-1815) : une référence centrale}

Dans la France de l'Ancien Régime, le cours d'histoire et de géographie d'Edme Mentelle à l'École royale militaire, fondée en 1751, qui va constituer une référence centrale parmi les modèles alternatifs au programme humaniste des collèges. Dans l'article «École militaire » de l'Encyclopédie, Jean-Baptiste Pâris de Meyzieu loue l'apparition de «cours publics » d'histoire - dans les collèges, elle était en effet le plus souvent enseignée sous forme de cours privés, dispensés aux élèves nobles, « en chambre $»^{26}$. Tout au long de sa carrière, comme précepteur de Mlle Fitz-James, professeur d'histoire et de géographie à l'École royale militaire, à l'École centrale des Quatre-Nations, professeur de géographie à l'École normale, puis comme auteur de manuels sous l'Empire et la Restauration, l'œuvre pédagogique de cet homme de réseau est marquée par une forte continuité27. Dans la plupart de ses manuels, il n'offre pas de longues narrations de l'histoire universelle similaires à celle de Millot, mais présente les cadres spatio-temporels dans lesquels ces événements se déroulent. Dans son cours de géographie à l'École normale de l'an III, il recommande l'usage de tableaux synoptiques, qui occupent une plus large place dans ses publications du Directoire et de l'Empire et reprennent le principe des tableaux analytiques de sa Géographie comparée, destinée à un public adulte sous l'Ancien Régime ${ }^{28}$. Dans l'édition de 1804 de son cours de géographie, Mentelle approuve de plus les tableaux chronologiques de Chrétien-Siméon Le Prévost d'Iray, professeur d'histoire à l'École centrale de la rue Saint-Antoine à Paris et donne une méthode pour les utiliser ${ }^{29}$.

À l'image des nombreux professeurs d'histoire des Écoles centrales qui mettent en avant l'importance de l'enseignement de la géographie, ou souhaitent même remplacer le cours d'histoire par un cours de géographie et de chronologie - c'est-à-dire introduire leurs élèves encore débutants aux cadres fondamentaux dans lesquels se déroulent les événements historiques - on attendrait, chez Pierre-Nicolas Chantreau et Pierre-Jean Massenet également, une référence à Mentelle, qui fait alors autorité ${ }^{30}$. Cette absence s'explique cependant par la position périphérique qu'occupent les deux professeurs de Strasbourg et d'Auch.

\section{Pierre-Nicolas Chantreau : un positionnement polémique et carriériste}

Pierre-Nicolas Chantreau fait une réponse fortement polémique à l'administration centrale et lui envoie un commentaire linéaire de la circulaire de Destutt de Tracy, dont il a recopié en marge les passages qu'il commente. Il rejette l'usage d'un livre élémentaire narratif et dit n'utiliser qu'un tableau des différentes notions qu'il faut maitriser pour pouvoir lire et écrire l'histoire correctement. Il élude la référence à Mentelle et 
Le Prévost d'Iray, qui enseignent tous deux dans des écoles parisiennes faisant figure de modèle, et il se présente comme le traducteur des Tablettes chronologiques de l'Anglais John Blair, recourant ainsi au modèle étranger pour faire concurrence à Mentelle sur un terrain commun, l'enseignement de l'histoire par tableaux synoptiques dans les Écoles centrales $^{31}$. La même année, il publie son propre tableau qui comporte trois grandes divisions, la chronologie, la géographie et l'économie politique ${ }^{32}$. Divisant les trois domaines en de nombres ramifications, il distingue par exemple dans l'économie politique le gouvernement, les opinions, les lois, l'état civil des individus, la langue et les usages. Chez cet ancien professeur de français en Espagne devenu fonctionnaire et journaliste durant les premières années de la Révolution, on peut voir une volonté de s'affirmer dans l'univers pédagogique et intellectuel du Directoire et d'utiliser le poste de professeur d'histoire comme un moyen d'ascension sociale ${ }^{33}$. En 1802, lors de la suppression des Écoles centrales, il publie un nouveau mémoire dans lequel il tente de promouvoir sa méthode pour les lycées en cours d'élaboration afin de poursuivre son ascension sous Napoléon ${ }^{34}$. L'ascension réussit. En 1808, l'année de sa mort, il est professeur d'histoire à l'École militaire de Saint-Cyr depuis un an, après avoir exercé à l'École militaire de Fontainebleau de 1803 à 1807 grâce à la bienveillance de François de Neufchâteau, avec qui Chantreau est suffisamment lié pour que le sénateur compose une ode concluant sa chronologie de l'histoire de France ${ }^{35}$. Le succès de Chantreau lui vaut d'ailleurs des attaques de la part d'un collaborateur de Mentelle, Conrad Malte-Brun, qui l'affronte encore une fois sur le terrain des modèles étrangers dans une recension de la Science de l'histoire, ouvrage dans lequel Chantreau a synthétisé ses vues ${ }^{36}$. Outre de nombreuses erreurs, il lui reproche de plagier la Géographie universelle de l'Allemand Anton Friedrich Büsching (1724-1793) et de faire passer la statistique (la science de l'État) pour une nouvelle science française alors qu'elle a été fondée par Gottfried Achenwall (1719-1772) à Göttingen dans les années $1760^{37}$.

19 Éluder la référence à Mentelle, se référer à des modèles étrangers, c'était, pour cet outsider des milieux culturels du Directoire, une manière de forcer son chemin parmi les personnalités établies. C'est en ce sens que l'on peut qualifier sa position de périphérique.

\section{Pierre-Jean Massenet : un enseignement républicain tourné vers la culture allemande}

Le cas de Pierre-Jean Massenet est également celui d'un professeur situé en périphérie du territoire français et se réclamant d'un modèle étranger pour rejeter les directives parisiennes. Cependant, le positionnement du professeur d'histoire de l'École centrale du Bas-Rhin est bien moins polémique. Le modèle allemand constitue chez lui une référence naturelle dans un lieu situé à la frontière entre deux cultures historiques :

«Je pense qu'il ne faut mettre entre les mains des élèves qu'un simple sommaire des faits les plus intéressans, de bonnes tablettes chronologiques où facilement ils puissent apercevoir et saisir la grande chaîne de l'histoire. Telles sont celles que le citoyen Koch membre de l'institut national a publiées à Strasbourg l'an VI. [...] Ces tablettes n'étant que d'histoire moderne, j'en donne d'autres à mes élèves pour l'histoire ancienne dans lesquelles je suis le plan de Millot dont les éléments d'histoire ancienne me paraissent supérieurs, quant à l'ordre des matières et à la netteté, à ses élémens d'histoire moderne, où les objets étant souvent trop mêlés il en naît de la confusion. Nous avons un ouvrage élémentaire du citoyen Koch, outre pour les tablettes chronologiques, bien préférable pour la régularité et la clarté du plan; c'est son tableau des révolutions de l'Europe en deux petits volumes ${ }^{38}$.» 
enet se réfère principalement aux ouvrages d'une personnalité strasbourgeoise, Christophe Guillaume Koch (1737-1813), élève de Jean-Daniel Schoepflin. À la mort de ce dernier, il reprend la direction de la célèbre École de diplomatie de Strasbourg en 1771. En 1787, Koch devient recteur de l'université de Strasbourg, puis député à l'Assemblée législative en $1791-1792^{39}$. Mentelle n'apparaît pas comme référence dans la lettre de Massenet tout simplement parce que Koch représente une référence plus évidente dans l'aire culturelle à laquelle appartient le professeur strasbourgeois. Si Millot fait également partie des références citées, c'est surtout pour les tablettes chronologiques qui accompagnent la partie consacrée à l'histoire ancienne de ses Élémens d'histoire générale ${ }^{40}$. Tandis que l'administration centrale se contente du livre de Millot faute de mieux, Massenet, pour l'histoire moderne, a trouvé à lui substituer les livres de Koch, qui connaissaient une réception importante dans l'espace germanophone ${ }^{41}$. Des ouvrages narratifs sont également mentionnés par Massenet (les élémens d'histoire générale de l'abbé Millot et le Tableau des révolutions de l'Europe de Christophe Guillaume Koch) mais ils ne sont pas utilisés par les élèves puisque Massenet ne «[met] entre [leurs] mains » que des tablettes chronologiques ${ }^{42}$. Selon toute apparence, ils servent seulement de guide et d'aide-mémoire au professeur.

S'ils tendent à présenter les modèles étrangers comme des innovations qu'ils importent en France, ni Chantreau ni Massenet ne promeuvent en réalité une méthode pédagogique radicalement nouvelle ou originale. S'ils rejettent l'idée d'un livre élémentaire narratif promue par le Conseil d'instruction publique, les modèles alternatifs ne manquent pas en France. Avec les professeurs parisiens Mentelle et Le Prévost d'Iray, nombreux sont les professeurs hors de Paris qui ont développé un enseignement plus axé sur la présentation tabulaire des connaissances, et même remplacé l'enseignement de l'histoire par celui de la géographie et de la chronologie afin de répondre aux attentes et au faible niveau de leurs élèves ${ }^{43}$. Pour comprendre le rôle des modèles étrangers, il ne faut donc pas penser en termes de tradition et d'innovation, mais s'intéresser aux enjeux géographiques et sociaux dans l'élaboration d'une pédagogie de l'histoire entre le centre et la périphérie de la République.

\section{Des formes de représentation complémentaires à des méthodes pédagogiques concurrentes}

\section{L'élémentation, une formation du jugement}

Dans le cadre d'une pédagogie des Lumières qui condamne la simple mémorisation et demande à l'élève de raisonner par lui-même, de comparer des données et de les unifier en un tout cohérent, Pierre-Jean Massenet associe la présentation tabulaire des connaissances dans la pédagogie de l'histoire à la notion de savoir élémentaire telle que l'ont définie D'Alembert et Condorcet. C'est ainsi que le professeur strasbourgeois associe le tableau à une méthode pédagogique émancipatrice concurrente de celle du Conseil d'instruction publique. Le professeur, en rejetant l'idée d'un livre élémentaire narratif, se distancie d'un enseignement qui transmettrait une version républicaine de l'histoire et défend une pédagogie qui transmet aux élèves les outils nécessaires pour forger leur propre vision, pour porter un jugement sur les différentes versions de l'histoire :

«Un bon livre élémentaire d'histoire aurait le précieux avantage de mettre de

l'uniformité dans l'enseignement. Mais s'il était parsemé de réflexions, toutes justes 
qu'elles fussent, il aurait l'inconvénient de prévenir le jugement des élèves, et d'en arrêter l'exercice qui cependant est, je le répète, le but le plus important que doive se proposer le professeur d'histoire, afin que l'habitude de bien observer le passé mette l'élève en état de bien juger le présent dans toutes les circonstances et de pressentir l'avenir ${ }^{44}$. » universités allemandes. Il reste cependant vague sur son modèle de référence et n'évoque qu'une méthode « usitée dans toutes les universités d'Allemagne » sans citer d'historien en particulier ${ }^{45}$. Pourtant, sa méthode est proche de celle de l'université de Göttingen, qui reste une initiative encore relativement isolée dans les pratiques universitaires allemandes des années 1790. Il n'en demeure pas moins que les historiens Johann Christoph Gatterer (1727-1799), fondateur du premier institut d'histoire dans l'espace germanophone, et son successeur August Ludwig Schlözer (1735-1809), ont en effet recouru à la tradition des tableaux synoptiques et s'en sont même parfois réclamés ${ }^{46}$. Dans la Présentation de son histoire universelle (Vorstellung seiner Universalhistorie), ouvrage didactique destiné à ses étudiants, Schlözer en définit l'usage en des termes qui font écho à la lettre de Massenet et soulignent l'apport des sens, de la vue d'ensemble, pour la raison:

«[...] ils [ces cahiers] sont seulement une présentation de mon histoire universelle, c'est-à-dire une présentation étayée par des preuves, du plan, de l'ordre et de l'étendue - certes assez importante, mais tout de même restreinte - selon lesquels je suis encore en mesure d'enseigner cette science dans des cours magistraux semestriels. Ils sont un fil rouge pour mes auditeurs : je leur épargne quelques semaines de séances préliminaires, je réduis le pénible travail de la prise de notes et je leur permets d'avoir facilement et à tout moment une vue d'ensemble sur mon cours ${ }^{47}$. "

La première moitié de la Présentation est une définition de l'histoire universelle : son objet est la manière dont l'homme s'est arraché à l'animalité pour tendre vers sa véritable nature, sa nature humaine, l'histoire universelle doit former un système et non se résumer à un agrégat de faits, elle doit prendre en compte tous les peuples et non pas seulement les plus célèbres, Hébreux, Grecs et Romains. La deuxième moitié de la Présentation est une sorte de chronologie commentée de l'histoire universelle. Schlözer se contente de poser les problèmes sans les développer, d'évoquer une foule d'exemples de manière très succincte et de citer un nombre limité de dates importantes. Les étudiants ne saisiront la véritable signification des questions posées et des événements cités que lorsqu'ils seront évoqués en cours. L'usage de la Présentation prévu par Schlözer est, on le voit, très similaire à celui que prévoit Massenet pour les tablettes chronologiques. Les faits mentionnés dans le tableau sont le point de départ d'une réflexion qui les lie entre eux, qui fait de l'agrégat de faits un système. Schlözer consacre de plus un développement important à la meilleure manière de retenir les dates : elles ne doivent pas être apprises par cœur ni en grande quantité, mais une réflexion sur l'écart de temps qui sépare deux moments importants de l'histoire universelle, sur les causes qui lient tel événement à tel autre, doit permettre une assimilation progressive et raisonnée d'une quantité de dates et de noms limitée à l'essentiel ${ }^{48}$. 
Les livres élémentaires de Schlözer et Koch, la méthode de Massenet mettent en avant, comme les encyclopédistes D'Alembert et Condorcet, la nécessité d'un apprentissage autonome des éléments par l'élève, les éléments ne devant pas tant être enseignés comme une somme statique de connaissances à mémoriser, que comme des principes et des données de base à maîtriser pour faire progresser une science. Ils se démarquent ainsi des Élémens d'histoire générale de Millot, qui, malgré leur titre, s'apparentent davantage à un abrégé présentant de manière concise l'état actuel des connaissances historiques ${ }^{49}$. Par référence à un modèle étranger présenté comme alternatif au modèle français, et dans la continuité de la valorisation des éléments par rapport à l'abrégé formulé par D'Alembert, Massenet en vient à associer une forme de représentation de l'histoire particulière à une méthode pédagogique précise. Si on compare la méthode promue par Massenet à celle que prescrit le Conseil d'instruction publique, on voit qu'elles ne sont pas radicalement opposées : dans les deux cas, la médiation du professeur est essentielle à une pédagogie de l'histoire réflexive et distanciée qui se distingue de la transmission par imprégnation de la pédagogie humaniste. Mais Massenet force le trait et associe la narration à un rôle passif, le tableau à un rôle actif de l'élève. Dans un contexte de politisation de l'enseignement de l'histoire, d'une quête du livre élémentaire vraiment républicain, on passe d'un modèle où différentes formes de représentation de l'histoire se prêtent à des usages multiples et complémentaires, à un modèle pédagogique qui tend à associer chaque forme de représentation de l'histoire à une méthode et des finalités pédagogiques particulières.

\section{Décomposer et classer : histoire, histoire naturelle, chimie et statistique}

«Savoir des élémens, ce n'est pas seulement connoître ce qu'ils contiennent, c'est en connoître l'usage, les applications, \& les conséquences [...]. » Écrire les éléments d'une science, c'est à la fois en définir les principes et énoncer les premières compétences qui doivent être transmises. Ce deuxième aspect de l'élémentation est caractéristique de la pédagogie développée par Pierre-Nicolas Chantreau, qui, dans ses tableaux synoptiques parfois placardés sur les murs de la classe, présente aux élèves la matrice à travers laquelle ils doivent glaner les faits au fil de leurs lectures, et à partir de laquelle ils doivent structurer chaque récit historique ${ }^{50}$. Ainsi, il donne à ses élèves des "Tablettes méthodiques pour lire l'histoire dans l'ordre qu'il convient de la lire et ramener toutes les histoires à un même plan quel que soit celui qu'aient suivi les historiens dont on fait usage.». Le plan est divisé en douze grands cadres: la chronologie, la géographie, l'histoire militaire, l'histoire politique, l'histoire des opinions religieuses et philosophiques, l'histoire des lois, l'histoire des usages, monnaies poids et mesures, l'histoire du commerce et navigation, l'histoire des finances, subsides et impôts, l'histoire des mœurs, l'histoire des découvertes et des arts, l'histoire de la littérature et de la langue parlée et écrite ${ }^{51}$. Le discours théorique sur l'histoire ne consiste pas, comme aujourd'hui, à en dégager les fondements philosophiques et esthétiques, mais à élaborer une structure, un plan de construction pour l'écriture de l'histoire ${ }^{52}$. Les nomenclatures mises au point par Chantreau sont conçues comme des plans de construction-types pour les récits historiques que le professeur fait écrire à ses élèves, substituant ainsi un ordre rationnel à l'ordre contingent de la découverte. Il rapporte par exemple à propos d'un tableau général de l'histoire universelle, dont il souligne la visée émancipatrice : 
«Je ne présente d'abord aux élèves que le canevas de mon tableau, et ils travaillent avec moi à en remplir le cadre et ici je cesse d'être professeur pour devenir le compagnon de leurs études. À la fin du tableau, ils ont acquis deux choses précieuses, celles de rédiger les faits et de n'en prendre que ce qui est important d'extraire ${ }^{53}$. »

De même, à propos de la mythologie égyptienne, grecque et romaine, il souligne la continuité entre savoirs savants et savoirs scolaires :

«Il m'arrive souvent de ne leur donner que le canevas des articles et rarement je m'en sais mauvais gré. C'est ainsi que j'ai d'un jeune homme un article Junon que bien des gens de lettres ne désavoueraient point ${ }^{54}$. »

Joseph Priestley, A Chart of Biography. Londres, Johnson, 1765.

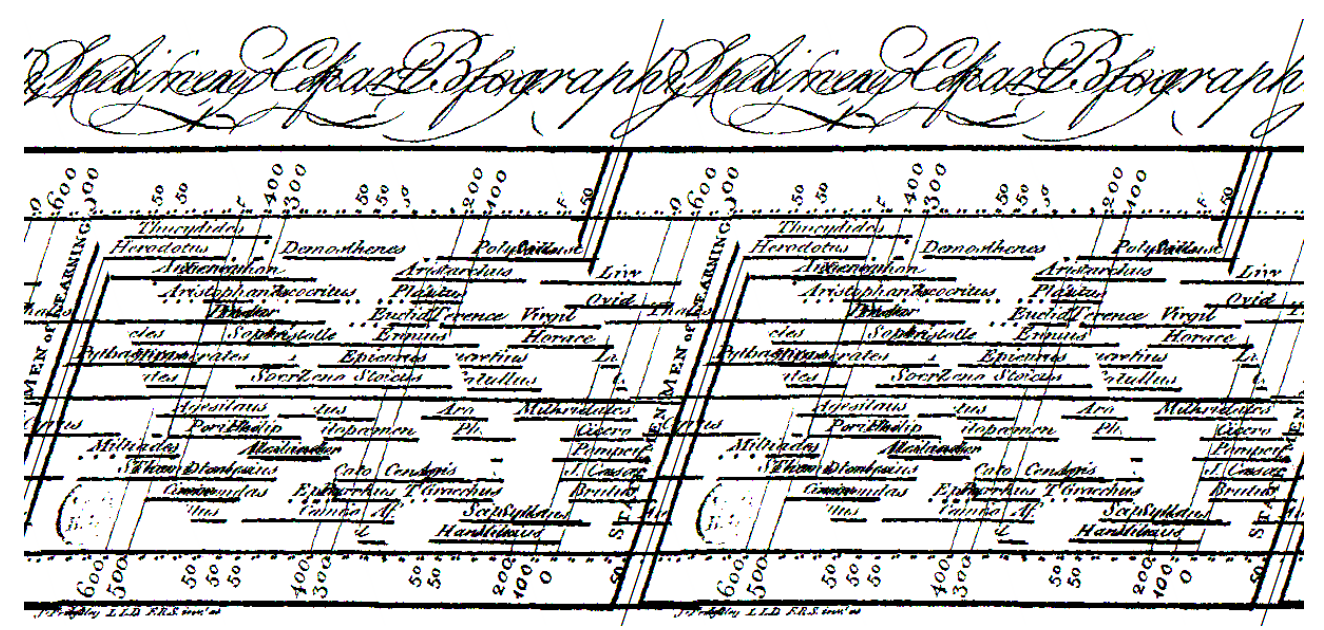

30 Le modèle anglais et la chimie servent ici de point de référence. C'est la traduction des tablettes chronologiques de John Blair en 1795 qui semble avoir été décisive pour Chantreau, puisqu'il commence à promouvoir les tableaux synoptiques à partir de ce moment-là et qu'ils ne sont pas présents dans son Manuel des Instituteurs publié un an plus tôt. Plus importante encore semble être la réception de la pédagogie de l'histoire de Joseph Priestley, dont il reprend l'idée des tables biographiques. Joseph Priestley (1733-1804), l'un des découvreurs de l'oxygène et défenseur du modèle phlogistique face à Lavoisier, écrit en effet dans ses Lectures on History (1788) que « la véritable histoire ressemble aux expériences faites à l'aide de la pompe à air, du condensateur ou de la machine électrique, qui montrent les opérations de la nature, et le Dieu de la nature luimême [...] et sont le travail et les matériaux de base des théories les plus générales et les plus utiles ${ }^{55}$. " Le lien avec la chimie est réaffirmé par Chantreau en 1804 lors de sa polémique avec Malte-Brun :

«J'ai mis en tableaux synoptiques toute la partie élémentaire de la chronologie, dont ils [ses détracteurs] n'ont pas dit un mot et pour cause : ce mode de présenter les élémens d'une science avant déjà été employé avec succès par de célèbres professeurs, et notamment par M. Fourcroy ${ }^{56}[\ldots]$. »

31 L'histoire, recueil des expériences humaines, doit devenir une science expérimentale qui décompose ses expériences en leurs composantes élémentaires et les ordonne dans un classement systématique. Constantin-François Volney expose, dans son cours à l'École normale de l'an III, une vision similaire :

«[...] si l'on avait un tableau exact de toutes les parties de chaque machine sociale, c'est-à-dire des habitudes, des opinions, des mœurs, des lois, du régime intérieur et 
extérieur de chaque nation, il serait possible d'établir une théorie générale de l'art

de composer ces machines morales ${ }^{57}[. .$.$] . »$ aux hommes les plus instruits et destinés aux fonctions publiques, Volney participe à la «promotion culturelle du citoyen» qui suit Thermidor et rapproche l'histoire de la statistique comme science de l'État ${ }^{58}$. Cette orientation vers la formation au service public est également présente chez Massenet: les tablettes chronologiques de Koch sont une version simplifiée de la chronologie qui accompagne son Abrégé de l'histoire des traités de paix entre les puissances de l'Europe depuis la paix de Westphalie (1796-1797) et destiné à la formation des diplomates ${ }^{59}$. À Göttingen, la systématisation des informations concernant l'État dans des tableaux est notamment définie par Gottfried Achenwall, le fondateur de la statistique dans l'espace germanophone, et reprise par August Ludwig Schlözer dans la deuxième moitié de sa carrière ${ }^{60}$. Cette orientation est également nette chez Chantreau, comme en témoignent sa nomination à l'École militaire de Fontainebleau en 1803 et les tableaux statistiques des départements de la République française qu'il publie dans le deuxième volume de sa Science de l'histoire en 1804, recommandées aux bibliothèques des lycées ${ }^{61}$. Émancipatrice en ce qu'elle forme au jugement autonome et à la constitution de leur propre version de l'histoire par les élèves, l'élémentation sur le modèle des sciences et de la statistique ne destine pas l'histoire à l'éducation populaire, mais à la formation des élites administratives et militaires. C'est à travers ces finalités que perdurent les tableaux synoptiques de Thermidor à l'Empire.

Sous le Directoire, Chantreau a réactualisé la tradition des tableaux synoptiques en l'associant à l'élémentation des savoirs pratiqués dans les sciences. Il a associé une pédagogie de l'élémentation au mode tabulaire de présentation des connaissances et il a légitimé sa démarche par la référence à des auteurs anglais afin de mieux s'opposer aux circulaires parisiennes et de mieux s'imposer face à des concurrents comme Edme Mentelle. C'est le choix stratégique de références anglaises qui, comme chez Massenet, a amené Chantreau à opposer deux démarches pédagogiques à travers le récit et le tableau, deux formes auparavant complémentaires.

\section{Conclusion}

Après l'enquête quantitative de Catherine Mérot ainsi que les recherches consacrées à des matières scolaires en général, cette étude de cas limitée à deux professeurs vient apporter un complément à la recherche sur les Écoles centrales ${ }^{62}$. Elle souligne le fait que chaque École a pu donner lieu à une réalisation particulière en fonction du contexte local. Elle explore surtout le rôle des modèles étrangers dans l'élaboration d'une pédagogie de l'histoire républicaine. Sur ce dernier point, elle invite à ne pas suivre naïvement le discours des professeurs, qui prétendent importer des méthodes nouvelles de l'étranger. En d'autres termes, elle invite à se dégager d'une vision trop réductrice des transferts culturels; elle explore les enjeux géographiques et sociaux de la reprise des modèles étrangers par des acteurs de la pédagogie républicaine, qui les utilisent pour se situer dans un contexte concurrentiel où de nouvelles références s'imposent tandis que d'autres sont éclipsées. Ce phénomène est le résultat d'une politisation de la pédagogie de l'histoire, d'une quête de livres élémentaires républicains qui instaure un rapport problématique à l'historiographie et à la pédagogie d'Ancien Régime et, par un jeu de références concurrentes, amène les professeurs à associer un mode précis de 
représentation de l'histoire à une méthode pédagogique émancipatrice. Pour l'enseignement de l'histoire, la spécificité du Directoire ne consiste pas en l'invention de nouvelles formes de représentation de l'histoire. Les diverses formes de récit ou de tableau historique utilisées ne se distinguent pas fondamentalement des formes qui avaient cours dans les siècles précédents ${ }^{63}$. La spécificité mise en avant dans cette étude réside au contraire dans le fait d'associer une forme de représentation particulière à une méthode pédagogique précise - d'opposer entre eux des modes de présentation des connaissances qui étaient auparavant utilisés en complémentarité. Elle s'explique par la présence de références étrangères, présentées comme une importation de méthodes innovantes, mais utilisées essentiellement comme alternatives aux références parisiennes. Le récit abrégé de l'histoire universelle, préconisé par l'administration centrale, est rejeté par deux professeurs à la périphérie de la République comme une méthode qui freine l'émancipation. Le tableau synoptique est légitimé par la référence étrangère et valorisé comme méthode pédagogique émancipatrice reposant sur l'élémentation du savoir.

Sans trancher sur les débats actuels, sur l'efficacité et les finalités pédagogiques de l'élémentation, cette étude de cas invite à se garder d'associer systématiquement telle forme de présentation des connaissances à telle méthode pédagogique, telle forme de représentation de l'histoire à telle volonté plus ou moins émancipatrice, comme l'a fait par exemple le didacticien Hans-Jürgen Pandel en opposant une tradition du catéchisme historique qui transmettrait une vision uniciste du passé, à l'innovation des récits historiques, dont la richesse esthétique permettrait une pluralité des interprétations ${ }^{64}$. Fortement politisée et affectée à la formation du citoyen à la fin du XVIIIe siècle, l'histoire ne peut certes manquer d'être transformée sur le plan esthétique et au niveau des contenus, mais elle l'est peut-être plus encore par les significations et finalités nouvelles attribués, par un jeu de références concurrentes, aux récits, tableaux, catéchismes et images hérités de l'Ancien Régime.

\section{NOTES}

1. Dominique JULIA, Les trois couleurs du tableau noir: La Révolution, Paris, Belin (Fondation de l'éducation), 1981 ; Marcel GRANDIÈRE, La formation des maîtres en France (1792-1914), Lyon, INRP (Éducation, histoire, mémoire) 2006, p. 15.

2. Jean-Luc CHAPPEY, "Les écoles de la Révolution : pour en finir avec la thèse de la table rase ", dans Michel Biard (dir.), La Révolution française. Une histoire toujours vivante, Paris, Taillandier, 2010, p. 331-343.

3. Cette question est partiellement évoquée dans Serge BIANCHI (dir.), Héros et héroïnes de la Révolution française, avec le concours de Bernard GAINOT et Pierre SERNA, Paris, Éditions du Comité des travaux historiques et scientifiques, 2012 et dans Philippe BOURDIN (dir.), La Révolution 1789-1871 : écriture d'une histoire immédiate, Clermont-Ferrand, Presses universitaires Blaise Pascal (Histoires croisées), 2008, notamment: Géraldine SOUDRI, "Les rois maudits ou la culture historique de la Terreur en 1791 », p. 87-110 (Voir également : Géraldine souDRI, Les rois maudits : 
de la culture historique révolutionnaire (1789-1791). Mémoire de maîtrise effectué à l'IHRF sous la direction de Pierre SERNA, 2004).

4. Martin S. STAUM, "L'Idéologie dans les Écoles centrales", dans François Azouvi (dir.), L'institution de la raison, Paris, Éditions de l'EHESS - Vrin, 1992, p. 163-196. Notamment pour la Troisième République, voir la bibliographe complète établie par Catherine MÉROT, dans sa thèse de l'École nationale des Chartes, La fréquentation et le recrutement des écoles centrales sous la Révolution, thèse de l'École des Chartes, 1985.

5. Un projet de recherche sous la direction de Arndt Brendecke, professeur à Ludwig-MaximilianUniversität de Munich, est consacré à ce genre historiographique, URL:http://www.sfbfrueheneuzeit.uni-muenchen.de/projekte/zusatz/HistorischeTabellenwerke/Index.html (dernière consultation 20.12.12, 15h45)

6. Je résume ici l'article de Arndt BRENDECKE, «Tabellenwerke in der Praxis der frühneuzeitlichen Geschichtsvermittlung ", dans Theo STAMMEN et Wolfgang E.J. WEBER (dir.), Wissenssicherung, Wissensordnung und Wissensverarbeitung. Das europäische Modell der Enzyklopädien, Berlin, AkademieVerlag, 2004, p. 157-189.

7. Isabelle SAINT-MARTIN, Voir, savoir, croire. Catéchismes et pédagogie par l'image au XIXe siècle, Paris, Champion (Histoire cuturelle de l'Europe), 2003, p. 178-185.

8. Chrétien-Siméon LE PRÉVOST D'IRAY, Tableau comparatif de l'histoire moderne, ouvrage adopté comme classique ou élémentaire par le gouvernement, pour les lycées, les écoles secondaires, etc. faisant suite au Tableau comparatif de l'histoire ancienne du même auteur. Par Ch. S. Le Prevost d'Iray, censeur des études du Lycée impérial, ci-devant professeur d'histoire aux Écoles centrales de Paris, Paris, Imprimerie impériale, 1804.

9. Michel foucault, Les mots et les choses. Une archéologie des sciences humaines, Paris, Gallimard, 1966, p. 89.

10. Johann Amos comenius, Orbis sensualium pictus, Prague, Trizonia, 1991.

11. Marcelle DENIS, Comenius, un pédagogue à l'échelle de l'Europe, chap : « La doctrine de Comenius est-elle sensualiste ?», Bern - Paris, Lang, 1992 ; Étienne KROTKY, Former l'homme, l'éducation selon Comenius (1592-1670), Paris, Publications de la Sorbonne, 1996; Theodor BRÜGGEMANN et HansHeino EWERS (dir.), Handbuch zur Kinder- und Jugendliteratur : von 1750 bis 1800, Stuttgart, Metzler, 1982, p. 1-19.

12. Michel fOUCAULt, Les mots et les choses, op. cit., p. 144-145.

13. Jean LE ROND D'ALEMBERT, Essai sur les éléments de philosophie ou sur les principes des connaissances humaines (1759), texte revu par Catherine KINTZLER, Paris, Fayard (Corpus des œuvres de philosophie en langue française), 1986 ; Id., « Discours Préliminaire des Éditeurs (Juin 1751) » et art. "Élémens des sciences » dans Encyclopédie, ou dictionnaire raisonné des sciences, des arts et des métiers, University of Chicago, ARTFL Encyclopédie Project (Spring 2011 Edition), Robert Morrissey (éd.) http://encyclopedie.uchicago.edu/.

14. Jean-Pierre SCHANDELER, "Les lieux de la rationalité de D’Alembert à Destutt de Tracy ", Recherches sur Diderot et sur l'Encyclopédie (En ligne), 25, 1998, mis en ligne le 04 août 2007, Consulté le 13 octobre 2012. URL : /index1281.html ; DOI : 10.4000/rde.1281; Alain TROUVÉ, La notion de savoir élémentaire à l'école. Doctrines et enjeux, préface de Michel FABRE, chap. : «Les livres élémentaires ", Paris, L'Harmattan (Savoir et formation), 2008, p. 141-143.

15. On la trouve notamment dans l'article "Élémens des sciences" de l'Encyclopédie : "Savoir des élémens, ce n'est pas seulement connoître ce qu'ils contiennent, c'est en connoître l'usage, les applications, \& les conséquences; c'est pénétrer dans le génie de l'inventeur, c'est se mettre en état d'aller plus loin que lui, \& voilà ce qu'on ne fait bien qu'à force d'étude \& d'exercice : voilà pourquoi on ne saura jamais parfaitement que ce qu'on a appris soi-même. Peut-être feroit-on bien par cette raison, d'indiquer en deux mots dans des élémens l'usage \& les conséquences des propositions démontrées. Ce seroit pour les commençans un sujet d'exercer leur esprit en 
cherchant la démonstration de ces conséquences, \& en faisant disparoître les vuides qu'on leur auroit laissés à remplir. Le propre d'un bon livre d'élémens est de laisser beaucoup à penser » Jean LE ROND D'ALEMBERT, art. «Élémens des sciences », dans Encyclopédie, op. cit., vol. 5, p. 496.

16. Nicolas de CONDORCET, « Rapport sur l'organisation générale de l'instruction publique » (avril 1792), dans Bronisław BАCZKO, Une éducation pour la démocratie: Textes et projets de l'époque révolutionnaire, Paris, Droz, 2000, p. 177-162 ; Alain TROUVÉ, La notion de savoir élémentaire à l'école. Doctrines et enjeux, op. cit., p. 123-211; Id., Penser l'élémentaire : la fin du savoir élémentaire à l'école?, Paris, L'Harmattan, 2010, p. 55-89 ; Dominique JULIA, Les trois couleurs du tableau noir : La Révolution, op. cit., p. 265-267 ; Catherine KINTZLER, "Éléments", dans Nicolas de CONDORCET, Écrits sur l'instruction publique. Vol. 1 : Cinq mémoires sur l'instruction publique, Charles Coutel et Catherine KINTZler (éd.), Paris, Edilig, (Les classes de la République) 1989, p. 253-261; id., Condorcet, L'instruction publique et la naissance du citoyen, Paris, Le Sycomore, 1984, p. 202-212.

17. «Rapport à la Convention du 7 brumaire an III (28 octobre 1794) sur les écoles primaires ", dans Marcel GRANDIÈRE, Rémi PARIS et Daniel GALLOYER (dir.), La formation des maitres en France, 1792-1914. Recueil de textes officiels. Lyon-Paris, INRP, 2007, p.15-16. Lakanal reprend une opposition déjà présente dans l'article «Élémens des sciences » de l'Encyclopédie, où D'Alembert dénonce « l'extrème disette où nous sommes de bons livres élémentaires, \& la foule de mauvais dont nous sommes chaque jour inondés. L'élève à peine sorti des premiers sentiers, encore frappé des difficultés qu'il a éprouvées, \& que souvent même il n'a surmontées qu'en partie, entreprend de les faire connoître \& surmonter aux autres ; censeur \& plagiaire tout ensemble de ceux qui l'ont précédé, il copie, transforme, étend, renverse, resserre, obscurcit, prend ses idées informes \& confuses pour des idées claires, \& l'envie qu'il a eu d'être auteur pour le désir d'être utile " Jean LE ROND D'ALEMBERT, art. «Élémens des sciences", dans Encyclopédie, op. cit., vol. 5, p. 496.

18. Thomas PRÜFER, Bildung der Geschichte: Friedrich Schiller und die Anfänge der modernen Geschichtswissenschaft, Köln, Böhlau, 2002.

19. Joseph LAKANAL, Rapport et projet de loi sur l'organisation des écoles primaires, présentés à la Convention nationale, au nom du Comité d'instruction publique, à la séance du 7 Brumaire, Paris, 28 octobre 1795, p. 7. Voir également art. "Livres scolaires", dans Ferdinand BUISson (dir.), Nouveau dictionnaire de pédagogie et d'instruction primaire, Paris, Hachette, 1911, édition électronique de l'IFÉ, URL: http://www.inrp.fr/edition-electronique/lodel/dictionnaireferdinand-buisson (dernière consultation 02/02/2012 13h32)

20. Christine LE BOZEC, «Le pas de clerc d'un centriste: Boissy d'Anglas et fructidor ", dans Philippe BouRdin et Bernard GAINOT (dir.), La République directoriale, Paris, Société des Études Robespierristes, 1998, p. 401-412.

21. AN, Conseil d'instruction publique. F/17/1011; Claude LE RAGOIS, Instruction sur l'histoire de France et romaine, augmentée jusqu'à la mort de Louis XVI, Amsterdam - Paris, Onfray, 1797 ; Philippe SERANE, Anecdotes romaines et françaises, ou parallèle des Romains et des Français suivi d'un Aperçu d'une éducation raisonnable, Paris, chez l'auteur, 1797.

22. Il a notamment revendiqué la paternité de certaines circulaires envoyées aux professeurs, voir Antoine-Louis-Claude DESTUTT DE TRACY, Premiers écrits 1789-1794. Sur l'éducation et l'instruction publique 1798-1805, Claude Joly (éd.), Paris, Vrin, 2011, p. 95, 149.

23. Matthias MEIRLAEN, «Philosophical history in the revolutionary school curriculum : ClaudeFrançois-Xavier Millot's Élémens d'histoire générale ", History of European ideas 36/3, 2010, p. 302-310.

24. Antoine-Louis-Claude DESTUTT DE TRACY, Premiers écrits 1789-1794. Sur l'éducation et l'instruction publique 1798-1805, op.cit., p.167-173; AN, Conseil d'instruction publique. Cahiers et autres enseignements admis par les professeurs d'histoire des Écoles centrales, an VI-VIII, F/17/1344/5. 
25. François HARTOG, L'Évidence de l'histoire: ce que voient les historiens, Paris, Gallimard (Folio Histoire), 2007.

26. Jean Baptiste Pâris de MEYZIEU, art. «École militaire », dans Encyclopédie, vol. 5, op. cit., p. 307-313.

27. Daniel NORDMAN (dir.), L'École normale de l'an III, Leçons d'histoire, de géographie, d'économie politique, Paris, Dunod (Librairie du bicentenaire de la Révolution française), 1994, p. 141-144.

28. Notamment: Edme MENTELlE, Cours complet de cosmographie, de géographie, de chronologie et d'histoire ancienne et moderne, divisé en 125 leçons, par le C. Mentelle, membre de l'Institut national et professé par lui à l'école des Quatre-Nations. Trois volumes in $8^{\circ}$, avec des Tableaux et un Atlas nouveau gravé par le C. Tardieu. Paris, Bernard, an XI (1802-1803) ; Edme MENTELLE, Géographie comparée, ou Analyse de la géographie ancienne et moderne des peuples de tous les pays et de tous les âges ; accompagnée de tableaux analytiques et d'un grand nombre de cartes, 7 vols., Paris, chez l'auteur, 1778. 29. Edme MENTELLE, La Géographie enseignée par une méthode nouvelle, ouvrage destiné aux écoles secondaires, $5^{\mathrm{e}}$ édition, Paris, chez l'auteur, 1804.

30. Catherine MÉROT, «La fréquentation des écoles centrales, un aspect de l'enseignement secondaire pendant la Révolution française. ", Bibliothèque de l'École des chartes, 145, 1987, p. 407-426, ici p. 416 : «[...] à Saint-Flour les professeurs d'histoire et de grammaire générale ont dû passer six mois à apprendre à lire à leurs élèves avant de pouvoir aborder toute autre activité [...] » et p. 417 ; Daniel NORDMAN (dir.), L'École normale de l'an III..., op. cit., p. 144-148, ici p. 144-145.

31. John BLAIR, Tables chronologiques qui embrassent toutes les parties de l'histoire universelle, année par année depuis la création du monde jusqu'en mil sept cent soixante huit publiées en anglois par John Blair, et traduites en françois par le citoyen Chantreau, qui les a continuées jusqu'à la paix conclue avec l'Espagne en 1795. Paris, Agasse, 1795.

32. Pierre-Nicolas $\mathrm{C}$ HANTREAU, Système analytique des notions qu'il faut acquérir pour connaître complètement l'histoire d'une nation, et le plan à suivre pour l'écrire. Par Chantreau, professeur d'Histoire à l'Ecole centrale du département du Gers, Paris, Debray, an VIII (1799-1800).

33. June K. BURTON, Napoleon and Clio : historical writing, teaching and thinking during the First Empire, Durham, Carolina academic press, 1979, p. 65-82.

34. «Deux motifs nous ont déterminé à publier ce développement; le premier pour le mettre sous les yeux du gouvernement, parce qu'il y a lieu de penser qu'à l'époque où il s'occupe de la réorganisation de l'instruction, il s'occupera aussi des méthodes ; [...]. Le second est l'espèce de proscription dont les chaires d'histoire ont parues menacées dans les Écoles centrales, proscription qui n'a pu être conçue que par la mauvaise idée que les instituteurs ont fait prendre de l'étude de l'histoire et du peu d'utilité dont ils ont rendu cette étude susceptible.» PierreNicolas CHANTREAU, De l'importance de l'étude de l'histoire et de la vraie manière de l'enseigner, d'après un nouveau plan présenté par tableaux qui contiennent les notions qu'il faut acquérir avant de se livrer à cette étude, et la méthode à suivre lorsqu'on s'y livre. Ouvrage aussi utile à ceux qui ont su qu'à ceux qui veulent savoir, et dont le développement est mis sous les yeux du Public et du Gouvernement. Par le citoyen Chantreau, auteur de la grammaire qui, en Espagne, sert pour l'étude de la langue française, traducteur des tablettes de John Blair, et Professeur d'histoire près l'École centrale du département du Gers, Paris, Deterville ; Auch, chez l'auteur et Lacaze An X (1801-1802), p. 8.

35. June K. BURTON, Napoleon and Clio..., op. cit., p. 73 ; Pierre-Nicolas CHANTREAU, Histoire de France abrégée et chronologique, depuis la première expédition des Gaulois jusques en septembre 1808, 2 vol., Paris, Bernard, 1808.

36. Pierre-Nicolas CHANTREAU, Science de l'histoire, contenant le système général des connaissances à acquérir avant d'étudier l'histoire et la méthode à suivre quand on se livre à ce genre d'étude, développée par tableaux synoptiques, 3 vol., Paris, Goujon, 1803-1806; Jean-Luc CHAPPEY, "Géographie et science de l'homme. Regards sur les enjeux scientifiques et politiques de voisinage et de 
distinction des savoirs ", dans Jean-Marc BESSE, Hélène BLAIS et Isabelle SURUN (dir.), Naissances de la géographie moderne (1760-1860), Paris, ENS Éditions, 2010, p. 195-217.

37. "Que les actes de naissance sont fâcheux pour les belles! Cette dame Statistique se donnait pour une petite Parisienne de quinze ans. Un de ses amans a même essayé de lui faire une généalogie grecque. Hélas! Au bout du compte, il se trouve que c'est une grosse Allemande, qui a près de quarante ans sur le dos. " Conrad MALTE-BRun, "Science de l'histoire, second extrait ", Journal des débats et des décrets, 8 octobre 1804 (16 vendémiaire an XIII), p. 2-4, ici p. 3.

38. AN, Conseil d'instruction publique. Cahiers et autres enseignements admis par les professeurs d'histoire des Écoles centrales, an VI-VIII, F/17/1344/5.

39. Jürgen voss, «Christoph Wilhelm Koch, homme politique et historiographe contemporain de la Révolution. », History of Europea ideas. 13/5, 1991 (numéro spécial : id. (dir.), Contemporary historians of the French Revolution) p. 531-543 ; id., Jean-Daniel Schoepflin (1694-1771) : un Alsacien de l'Europe des Lumières, traduit par Bernard Rolling, Strasbourg, Société savante d'Alsace, 1999.

40. "Table de géographie ancienne, pour faciliter la compréhension de cet ouvrage ", dans François-Xavier-Claude міLLот, Élémens d'histoire générale, Première partie, Histoire ancienne. t. 1, édition nouvelle, augmentée, Paris, Durand, 1778 [1772].

41. Réception qui lui vaudra une traduction berlinoise: Christoph Wilhelm косн, Gemählde der Revolutionen in Europa : seit dem Umsturze des Römischen Kaiserthums im Occident, bis auf unsere Zeiten, mit einer Einleitung in die Geschichte, chronologischen und genealogischen Tabellen Landkarten und einem vollständigen Register, traduit par Johann Daniel SANDER, avec une préface de Friedrich BUCHHOLZ, 3 vol., Berlin, Sander, 1807-1809.

42. Christophe Guillaume косн, Tableau des révolutions de l'Europe, depuis le bouleversement de l'Empire d'Occident jusqu'à nos jours, Lausanne - Strasbourg, Bauer \& $C^{\text {ie }}, 1771$.

43. Dans sa lettre du 24 frimaire an VIII (19 décembre 1799), Raymond, professeur d'histoire dans le département du Mont-Blanc, va même jusqu'à demander le «remplacement de la chaire d'histoire par une chaire de géographie et de chronologie » AN, Conseil d'instruction publique. Cahiers et autres enseignements admis par les professeurs d'histoire des Écoles centrales, an VIVIII, F/17/1344/5.

44. AN, Conseil d'instruction publique. Cahiers et autres enseignements admis par les professeurs d'histoire des Écoles centrales, an VI-VIII, F/17/1344/5.

45. Ibid.

46. Martin GIERL, Geschichte als präzisierte Wissenschaft: Johann Christoph Gatterer und die Historiographie des 18. Jahrhunderts im ganzen Umfang, Stuttgart - Bad Cannstatt, FrommannHolzboog (Fundamenta historica), 2012 ; Martin PETERS, Altes Reich und Europa: der Historiker, Statistiker und Publizist August Ludwig (v.) Schlözer (1735-1809), München, Lit, 2005 ; Arndt BRENDECKE, «Tabellenwerke in der Praxis der frühneuzeitlichen Geschichtsvermittlung », op. cit., p. 163.

47. August Ludwig SCHLÖZER, Vorstellung seiner Universalhistorie, 2 vol. Göttingen - Gotha, Dieterich, 1772-1773, préface (Vorrede) n. p. . Je souligne et je traduis. « [...] sie [diese Bogen] sind blos eine Vorstellung meiner Universalhistorie, das ist, eine mit Beweisen belegte Vorstellung des Plans, der Ordnung, und des zwar etwas erweiterten, mit aber immer noch engen Umfangs, wonach ich diese Wissenschaft in halbjährigen Vorlesungen noch zur Zeit vorzutragen im Stande bin. Sie sind ein Leitfaden für meine Zuhörer, denen ich dadurch ein paar Wochen Prolegomenen erspare, die Mühe des Nachschreibens vermindere, und die beständige Überschauung des Ganzen erleichtere."

48. Ibid., p. 51-53.

49. À propos du terme éléments, Chantreau distingue les éléments d'histoire, résumés au nombre desquels comptent les Élémens de Millot, des éléments de l'histoire, matrice de la science 
historique. AN, Conseil d'instruction publique. Cahiers et autres enseignements admis par les professeurs d'histoire des Écoles centrales, an VI-VIII, F/17/1344/5.

50. Ibid.

51. Ibid.

52. C'est ce qu'a clairement formulé Martin Gierl à propos de l'œuvre théorique de Gatterer. Martin GIERL, Geschichte als präzisierte Wissenschaft..., op. cit., p. 37.

53. AN, Conseil d'instruction publique. Cahiers et autres enseignements admis par les professeurs d'histoire des Écoles centrales, an VI-VIII, F/17/1344/5.

54. Ibid.

55. "Real history resembles the experiments made by the air pump, the condensing engine or electrical machine, which exhibit the operations of nature, and the God of nature himself [...] and are the ground work and materials of the most extensive and useful theories. " Robert E. SCHOFIELD, The Enlightenment of Joseph Priestley: A Study of His Life and Work from 1733 to 1773, University Park, Pennsylvania State University Press, 1997, p. 165.

56. Pierre Nicolas chantreau, «Réponse de M. Chantreau aux articles insérés par $M$. MATTEBRUN (sic!) dans le Journal des Débats, sur l'ouvrage intitulé : la Science de l'Histoire. ", La revue, (ou Décade) Philosophique, littéraire et politique, par une société de gens de lettres. An XIII, $1^{\mathrm{er}}$ trimestre (Vendémiaire, Brumaire, Frimaire), p. 471.

57. François-Constantin VOLNEY, Leçons d'histoire à l'École normale de l'an III. Quatrième leçon, dans Daniel Nordman (dir.), L'École normale de l'an III..., op. cit., p. 113.

58. Bronisław BACZKO, «La constitution de l'an III et la promotion culturelle du citoyen. », dans François Azouvi (dir.), L'institution de la raison, op. cit., p. 21-37.

59. Christophe-Guillaume косн, Abrégé de l'histoire des traités de paix entre les puissances de l'Europe depuis la paix de Westphalie, 4 vol. Bâle, Decker ; Paris, Onfroi - Pougens ; Strasbourg, König Levrault ; Leipzig - Leo, 1796-1797.

60. Arndt BRENDECKE, «Tabellenwerke in der Praxis der frühneuzeitlichen Geschichtsvermittlung », op. cit., p. 163-173.

61. Pierre-Nicolas chantreau, Science de l'histoire, vol. 2, Géographie: Europe, Paris, Goujon fils, 1804.

62. Catherine MÉROT, La fréquentation et le recrutement des écoles centrales sous la Révolution, op. cit. ; Dominique JULIA (dir.), Atlas de la Révolution française, t. 2 : L'Enseignement 1760-1815, Paris, EHESS, 1987, p. 40-45 ; Martin S. STAUM, "L'Idéologie dans les Écoles centrales », op. cit., p. 163-196; Marcel GUY, «L'enseignement de l'histoire dans les écoles centrales ", Annales historiques de la Révolution française, 53, 1981, p. 89-122.

63. Hormis pour les catéchismes historiques, peu utilisés dans les Écoles centrales, et dont Annie Bruter a démontré l'importance à la fin du XVII ${ }^{\mathrm{e}}$ siècle : Annie BRUTER, L'histoire enseignée au grand siècle : naissance d'une pédagogie, Paris, Belin, 1997.

64. Hans-Jürgen PANDEL, Historik und Didaktik: das Problem der Distribution historiographisch erzeugten Wissens in der deutschen Geschichtswissenschaft von der Spätaufklärung zum Frühhistorismus (1765 - 1830), Stuttgart - Bad Cannstatt, Frommann-Holzboog (Fundamenta historica), 1990. 


\section{RÉSUMÉS}

Le cas des deux professeurs d'histoire des Écoles centrales d'Auch et Strasbourg, en périphérie du territoire, permet d'éclairer le rôle des modèles étrangers dans l'élaboration d'une pédagogie dite républicaine. Dans le contexte d'une réception problématique de l'héritage de l'Ancien Régime, tous deux défendent l'utilisation de manuels scolaires non narratifs, présentant l'histoire sous forme de tableaux synoptiques et se rattachant à une conception émancipatrice de l'élémentation des savoirs. C'est la référence aux modèles pédagogiques étrangers, allemands et anglais, présentée comme une innovation importée, qui justifie la remise en question des références centrales (le Conseil d'Instruction publique et les Écoles centrales parisiennes) et qui amène les deux professeurs à associer un mode de présentation des connaissances particulier à une pédagogie émancipatrice.

The case of two history teachers in the Écoles centrales of Auch and Strasbourg, located in the periphery of the French territory, sheds a new light on the role of the German and the English models in the development of a so-called republican pedagogy. In the context of the problematic reception of Old Regime history books, both teachers recommend using non narrative textbooks which represent History in synoptic tables and are linked to an emancipating understanding of elementary knowledge. Through the reference to German and English models, presented as an import of innovative methods, they justify their questioning of the central references (the Conseil d'Instruction publique and the parisian Écoles centrales) and propose to combine together a specific way of representing knowledge and an emancipating pedagogy.

\section{INDEX}

Keywords : Ecoles centrales, pedagogy, history, historiography, tables, cultural transfert, popularisation, textbooks

Mots-clés : Écoles centrales, pédagogie, histoire, historiographie, tableau, transferts culturels, éléments, élémentation, manuels scolaires 